\title{
Treatment Outcomes of Hepatocellular Carcinoma Patients who underwent Stereotactic Body Radiotherapy
}

\author{
Paytai Rordlamool, M.D. ${ }^{1}$, Kittipitch Bannangkoon, M.D. ${ }^{2}$, Naichaya Chamroonkul, M.D. ${ }^{3}$ \\ ${ }^{1}$ Therapeutic Radiology and Oncology Unit, Department of Radiology, Faculty of Medicine, Prince of Songkla University, \\ Hat Yai, Songkhla 90110, Thailand. \\ ${ }^{2}$ Interventional Radiology Unit, Department of Radiology, Faculty of Medicine, Prince of Songkla University, Hat Yai, Songkhla \\ 90110, Thailand. \\ ${ }^{3}$ Gastroenterology \& Hepatology Unit, Department of of Internal Medicine, Faculty of Medicine, Prince of Songkla University, \\ Hat Yai, Songkhla 90110, Thailand. \\ Received 30 September 2021 • Revised 13 December 2021 • Accepted 27 December 2021 • Published online 3 February 2022
}

\begin{abstract}
:
Objective: To evaluate the prognosis of patients with hepatocellular carcinoma (HCC) treated with stereotactic body radiotherapy (SBRT).
\end{abstract}

Material and Methods: A retrospective review was performed of the institutional medical records of patients treated with SBRT. The selection criteria were as follows: unresectable HCC confirmed via pathologically, or through imaging, a Karnofsky Performance Status score $>60$, any Barcelona Clinic Liver Cancer stage, a Child-Pugh score $\leq 8$, and an uninvolved liver volume $>700 \mathrm{~cm}^{3}$. The primary outcomes were local tumor control, overall survival, and progressionfree survival rates. The secondary outcomes were acute adverse events; including general, gastrointestinal, or hepatic disorders; decreased complete blood count; and increased liver function test results.

Results: We included 27 HCC patients treated with SBRT; from August 2013 to October 2019. Moreover, 55.6\% of patients had received previous treatments for their SBRT-treated lesions. Additionally, the median volume of internal target volume was $40 \mathrm{~cm}^{3}$, the median uninvolved liver volume was $1,162 \mathrm{~cm}^{3}$, and the median radiation dose was 40 Gray: given in five fractions. The 1- and 2-year local tumor control rates were both $79.5 \%$. The 1- and 2-year overall survival rates were $58.8 \%$ and $27.6 \%$, respectively, and the median survival was 13 months. There were no grade 4 or 5 acute adverse events observed at initiation of treatment or during the follow-up period. Thirteen percent of patients experienced grade 3 acute adverse events. Three patients experienced radiation-induced liver disease.

Contact: Paytai Rordlamool, M.D.

Therapeutic Radiology and Oncology Unit, Division of Radiology, Faculty of Medicine,

Prince of Songkla University, Hat Yai, Songkhla 90110, Thailand.

E-mail: drpaytai@yahoo.com

(c) 2022 JHSMR. Hosting by Prince of Songkla University. All rights reserved.

This is an open access article under the CC BY-NC-ND license

(http://www.jhsmr.org/index.php/jhsmr/about/editorialPolicies\#openAccessPolicy).
J Health Sci Med Res 2022;40(5):519-531 doi: 10.31584 /jhsmr.2022861 www.jhsmr.org 
Conclusion: SBRT can be an effective local treatment for hepatocellular carcinoma in carefully selected patients.

Keywords: adverse events; hepatocellular carcinoma; radiotherapy; stereotactic body radiotherapy; treatment outcome

\section{Introduction}

In Thailand, hepatobiliary cancer is the most common cancer among males, and the second most common cancer among females. Most patients with hepatobiliary cancer in southern Thailand are diagnosed with hepatocellular carcinoma (HCC), with a mean annual age-standardized incidence rate of 9.1 per 100,000 population. ${ }^{1}$ Although, hepatic resection, liver transplantation, thermal ablation, or percutaneous ethanol injection can be used to cure early-stage HCC, most patients present to physicians with late-stage HCC; hence, curative-intent treatment cannot be administered. ${ }^{2-4}$ Moreover, our previous studies have shown that although $27.1 \%$ of patients can be treated, only $1.5 \%$ of patients are specifically suitable for curativeintent treatments. Thus, palliative treatments are essential, because untreated patients face poor prognosis with a median survival of only 1.6 months. $^{3}$

Transarterial chemoembolization (TACE) is the most common palliative treatment, with a 2-year overall survival rate of $41 \%{ }^{5}$ and a 1 -year local tumor control rate of $69.9 \%$. Additionally, targeted therapy with sorafenib leads to a 1-year overall survival rate of $44 \%{ }^{7}$ Finally, stereotactic body radiotherapy (SBRT) has a 1-year overall survival rate of $55 \%{ }^{8}$ and a 1 -year local control rate of $87 \%{ }^{8}$ or 2 -year local control rate of $90-91 \% .^{9,10}$

In the past, radiotherapy has had a limited role in treating $\mathrm{HCC}$, due to concerns regarding radiation-induced liver disease (RILD). However, recent advances in treatment technology and tumor localization techniques have enabled the safe delivery of radiation to a partial volume of the liver. Specifically, there are four radiotherapy modalities for the treatment of HCC: three-dimensional conformal radiotherapy (3D-CRT), intensity-modulated radiotherapy, volumetric-modulated arc therapy (VMAT), and SBRT. SBRT differs from 3D-CRT in its use of a higher dose per fraction, and enables a more accurate identification of the tumor location. Attention has been dedicated to developing SBRT, because of its ability to spare normal liver tissues and its reduced mean liver dose (MLD) as well as the lack of success in conventional fractionation radiation. ${ }^{11}$ SBRT may result in more positive outcomes than conventional radiotherapy due to its ability to induce endothelial cell damage and an increased immune response. ${ }^{12}$

Although, there are many published studies on the outcomes of HCC patients treated with SBRT, most of them come from countries with advanced medical resources, and therefore HCC of different etiologies ${ }^{2,13-15}$, which could lead to different prognoses. ${ }^{16,17}$ However, there is a lack of data from Thailand regarding the outcomes of SBRT treatment in patients with HCC. Therefore, in this study, we aimed to determine the SBRT treatment outcomes in terms of local tumor control, overall survival, and progression-free survival rates as well as acute adverse events in Thailand. These findings are crucial for guiding future research on improving palliative treatments for HCC patients.

\section{Material and Methods}

A review of medical records and the treatmentplanning software database; from August 2013 to October 2019, was performed to identify HCC patients treated with SBRT. The selection criteria were as follows: unresectable HCC confirmed via pathologically, or through imaging, a Karnofsky Performance Status (KPS) score $>60$, any Barcelona Clinic Liver Cancer (BCLC) stage, unsuitable 
for or refractory to other liver-directed therapy, a ChildPugh score (CPS) $\leq 8$, no treatment with medication that increases the risk of bleeding at least 2 weeks before the start of SBRT, adequate bone marrow and organ function, $\leq 2$ discrete lesions, an uninvolved liver volume $>700 \mathrm{~cm}^{3}$ and no extrahepatic disease.

The baseline characteristics of each patient were recorded (Table 1). The KPS score, CPS, BCLC stage, alpha-fetoprotein (AFP) level, and acute SBRT-related complications were recorded at the time of consultation, in the first and second weeks of SBRT treatment, and at 1 and 3 months after SBRT treatment. After 3 months of SBRT treatment, the KPS score, CPS, BCLC stage, and AFP level will be recorded every 3 months until patient loss to follow up. For medical records from outside our unit, Eastern Cooperative Oncology Group (ECOG)0, ECOG1, and ECOG2 were defined as KPS scores of 90-100, 70-80 and $50-60$, respectively. ${ }^{18}$ This study was approved by the institutional human research ethics committee. The need for informed consent was waived; as this is a retrospective chart review.

Patients were immobilized in the arms-up position in the Body Pro-Lok ${ }^{\mathrm{TM}}$ System for SBRT (CIVCO Radiotherapy); all patients were treated with free breathing, and abdominal compression. Image acquisition was performed using the Philips Brilliance Big Bore four-dimensional computed tomography (4DCT) in the arterial and portal phase with a slice thickness of $0.2 \mathrm{~cm}$ (Phillips Healthcare). Additionally, respiratory phase was classified using the respiratory bellows gating system (Phillips Healthcare). Maximal intensity projection and phase 75 respiration were created for further treatment planning purposes. Knee and feet support devices were supplied for patient comfort. All patients were treated without fiducial marker implantation in the tumor.

Gross tumor volume was defined as an enhancing tumor in the arterial phase, and/or washed out in the portal phase. Contouring was performed in the maximal intensity projection or portal phases, to create an internal target volume (ITV) to account for tumor motion, and the planning target volume (PTV) was expanded $0.7-1.0 \mathrm{~cm}$ in all directions from the ITV. No clinical target volume was contoured. At minimum, all organs at risk, within irradiated areas and $2 \mathrm{~cm}$ above and below the treatment plane, were contoured. Treatment planning was performed primarily in phase 75 of the arterial or portal phase, using the Eclipse ${ }^{\mathrm{TM}}$ treatment planning system version 10.0.42 (Varian Medical Systems). The dose was 27.5-50 Gray (Gy) in 5 fractions. The preferred inter-fraction interval was $48 \mathrm{~h}$. The prescription dose ideally encompassed $95.0 \%$ of the PTV. Furthermore, the maximal allowed dose within the PTV was $150.0 \%$, and 30 Gy isodose was made to be conformal as much as possible. This study is based on the prescription dose and normal tissue constraints listed in the Radiation Therapy Oncology Group 1,112 protocols. $^{19}$

Most patients were treated with five fractions of SBRT on alternate days. Treatment was administered by VMAT using the Truebeam ${ }^{\mathrm{TM}}$ STx system's linear accelerator (Varian Medical Systems). For each fraction, image-guided radiotherapy was administered using onboard imaging, and a cone-beam computed tomography (CT) system as well as previously retained lipiodol staining, or liver contouring and adjacent organ comparison.

Lesion responsiveness to treatment was classified according to the Modified Response Evaluation Criteria in Solid Tumors (mRECIST) ${ }^{20}$, via CT or magnetic resonance imaging. Local tumor control for SBRT-treated lesions was determined as one of the following: complete response, partial response, or stable disease. Local progression was determined as progressive disease. Tumors without arterial enhancement were classified using the RECIST criteria. ${ }^{21}$ An interventional radiologist specializing in HCC assessed tumor response every 3 months after completion of SBRT treatment. Local tumor control was defined as 
the time from initial SBRT administration to local tumor progression. Overall survival was defined as the time from initial SBRT administration to death from any cause. Progression-free survival was defined as the time from initial SBRT administration to disease progression (according to mRECIST or RECIST criteria) or patient death from any cause. The SBRT-related acute adverse events were graded according to the Common Terminology Criteria for Adverse Events version $5 .^{22}$ Clinical follow-up was performed during the first and second weeks of SBRT treatment, 1 month after SBRT treatment, 3 months after SBRT treatment, and then at 3- to 4-month intervals until patient death or loss to follow-up. Generally, imaging followup was performed at 3-month intervals post-treatment.

The primary endpoints were local tumor control rate, overall survival rate, and progression-free survival rate. Local tumor control rate was computed using competing risk analysis; we defined death from any cause as a competing event. Overall survival and progression-free survival were analyzed, and are presented using Kaplan-Meier curves. The secondary endpoint was SBRT-related acute toxicity from initial presentation to 3 months post-treatment. Post hoc analysis of stratified uninvolved liver volume and other factors associated with overall survival were computed using a Cox proportional hazards regression model. A p-value $<0.050$ was considered significant. Statistical analyses were performed using RStudio program, version 1.4.1106 and Stata 17.0.

\section{Results}

This study took place from August 2013 to October 2019, and included 27 HCC patients treated with SBRT. All baseline characteristics are listed in Table 1. The median age was 64 (interquartile range (IQR), 57-69) years, with. most patients having a KPS score of 70 . The most common cause of cirrhosis was hepatitis B virus (HBV) infection (40.7\%), and the most common CPS at initiation of treatment was 5 (48.2\%). Most patients were classified as BCLC stage C (92.6\%). Additionally, the most common reason for SBRT treatment was the presence of lesions that were untreatable with other types of liver-directed therapy $(70.4 \%)$. Moreover, $55.6 \%$ of patients underwent other treatment modalities (i.e., TACE, thermal ablation, or radiofrequency ablation) before the initiation of SBRT treatment. The median AFP level at initiation of SBRT was 63 (IQR, 9-375) IU/mL. The median longest tumor diameter before the initiation of SBRT treatment was 3 (IQR, 2-6) $\mathrm{cm}$, and the median total ITV was 40 (IQR, 18-161) $\mathrm{cm}^{3}$. The uninvolved liver volume had a median value of 1,162 (IQR, 942-1,303) $\mathrm{cm}^{3}$, and the median MLD was 14 Gy (IQR, 9-15). The median total treatment time was 9 (IQR, 8-13) days. The median SBRT dose was 40 (IQR, 38-45) Gy, and most of the patients (96.3\%) were administered SBRT in 5 fractions on alternate days.

Table 1 Baseline characteristics [data presented as No. (\%), unless otherwise indicated]

\begin{tabular}{|c|c|}
\hline Characteristic & \\
\hline \multicolumn{2}{|l|}{ Gender } \\
\hline Male & $24(88.9 \%)$ \\
\hline Female & $3(11.1 \%)$ \\
\hline Median age (IQR), years & $64(57-69)$ \\
\hline \multicolumn{2}{|l|}{ KPS at time of SBRT } \\
\hline 100 & $2(7.4 \%)$ \\
\hline 90 & $6(22.2 \%)$ \\
\hline 80 & $5(18.5 \%)$ \\
\hline 70 & $13(48.2 \%)$ \\
\hline 60 & $1(3.7 \%)$ \\
\hline \multicolumn{2}{|l|}{ Cause of cirrhosis } \\
\hline HBV infection & $11(40.7 \%)$ \\
\hline HCV infection & $8(29.6 \%)$ \\
\hline Non-viral & $8(29.6 \%)$ \\
\hline \multicolumn{2}{|c|}{ Child Pugh score at time of SBRT } \\
\hline 5 & $13(48.2 \%)$ \\
\hline 6 & $10(37.0 \%)$ \\
\hline 7 & $2(7.4 \%)$ \\
\hline 8 & $2(7.4 \%)$ \\
\hline \multicolumn{2}{|c|}{ BCLC stage at time of SBRT } \\
\hline 0 & $0(0.0 \%)$ \\
\hline$A$ & $1(3.7 \%)$ \\
\hline $\mathrm{B}$ & $1(3.7 \%)$ \\
\hline C & $25(92.6 \%)$ \\
\hline
\end{tabular}


Table 1 (continued)

\begin{tabular}{|c|c|}
\hline \multicolumn{2}{|l|}{ Characteristic } \\
\hline \multicolumn{2}{|l|}{ Reasons for the indication of SBRT } \\
\hline $\begin{array}{l}\text { Lesion unsuitable or a contraindication } \\
\text { for other LDT }\end{array}$ & $19(70.4 \%)$ \\
\hline Lesion refractory to other LDT & $7(25.9 \%)$ \\
\hline Patient refused other LDT & $1(3.7 \%)$ \\
\hline \multicolumn{2}{|l|}{ Lipiodol stained lesion } \\
\hline Yes & $10(37.0 \%)$ \\
\hline No & $17(63.0 \%)$ \\
\hline \multicolumn{2}{|l|}{ Prior LDT to SBRT treated lesion } \\
\hline Yes & $15(55.6 \%)$ \\
\hline No & $12(44.4 \%)$ \\
\hline $\begin{array}{l}\text { Median AFP level at time of SBRT (IQR), } \\
\text { IU/mL }\end{array}$ & $63(9-375)$ \\
\hline Median longest tumor diameter (IQR), cm & $3(2-6)$ \\
\hline Median total ITV volume (IQR), $\mathrm{cm}^{3}$ & $40(18-161)$ \\
\hline Median PTV volume (IQR), $\mathrm{cm}^{3}$ & $136(75-408)$ \\
\hline Median uninvolved liver volume (IQR), $\mathrm{cm}^{3}$ & $1,162(942-1,303)$ \\
\hline $\begin{array}{l}\text { Median mean liver minus ITV dose (IQR), } \\
\text { Gy }\end{array}$ & $14(9-15)$ \\
\hline Median total treatment time (IQR), days & $9(8-13)$ \\
\hline \multicolumn{2}{|l|}{ SBRT fractionation } \\
\hline 50 Gy / 5 fractions & $5(18.5 \%)$ \\
\hline 45.5 Gy / 7 fractions & $1(3.7 \%)$ \\
\hline 45 Gy/ 5 fractions & $2(7.4 \%)$ \\
\hline 40 Gy / 5 fractions & $12(44.4 \%)$ \\
\hline 35 Gy / 5 fractions & $2(7.4 \%)$ \\
\hline 32.5 Gy / 5 fractions & $2(7.4 \%)$ \\
\hline 30 Gy / 5 fractions & $3(11.1 \%)$ \\
\hline
\end{tabular}

†Due to rounding of decimals, totals can add up to be less or greater than $100 \%$

$A F P=a l p h a$ fetoprotein, BCLC=Barcelona liver cancer clinic, Gy=Gray, $\mathrm{HBV}=$ hepatitis $B$ virus, $\mathrm{HCV}=$ hepatitis $C$ virus, $I Q R=$ interquartile range, ITV=internal target volume, KPS=Karnofsky performance status, LDT=liver directed therapy, SBRT=stereotactic body radiotherapy

With a median follow-up time of 12 months, the 1- and 2-year local tumor control rates were both $79.5 \%$ (Figure 1). After 6.3 months, there was no event of local tumor progression for all patients. In regards to the 1-year local tumor control response, complete response was seen in $35.7 \%$, partial response in $21.4 \%$, and stable disease in $28.6 \%$ of the cases. The 1- and 2-year overall survival rates were $58.8 \%(95 \% \mathrm{Cl}, 43-81 \%)$ and $27.6 \%(95 \%$ $\mathrm{Cl}, 14-54 \%$ ) (Figure 2), respectively, and the median survival was 13 months (IQR, 2-75). The 1- and 2-year progression-free survival rates were $52.2 \%(95 \% \mathrm{Cl}, 35-$ $78 \%)$ and $35.8 \%(95 \% \mathrm{Cl}, 18-70 \%)$ (Figure 3), respectively. Patients with progressive disease after SBRT experienced poorer overall survival than those with controlled disease, although the difference was not statistically significant (hazards ratio, 2.02; 95\% Cl, 0.656.23; $p$-value $=0.222$ ). The median time from progressive disease to death was 161 days (IQR, 110-568.5).

Regarding adverse events (Table 2 and 3), 1 of the 27 patients had incomplete follow-up at 3 months postSBRT. Additionally, there were no grade 4 or 5 acute adverse events observed; either at initial presentation or during follow-up. The most common acute adverse events during the first week, during the second week, after 1 month, and after 3 months of SBRT were: gastrointestinal (Gl) disorders, increased aspartate aminotransferase levels, decreased platelet counts and decreased platelet counts, respectively. The proportion of patients who experienced grade 3 acute adverse events during the first 3 months of the follow-up period were as follows: general disorders, 7.4\%; Gl disorders, 7.4\%; abnormal complete blood count, 18.5\%; and abnormal liver function test result, $29.6 \%$. One patient with an ITV and uninvolved liver volume of 632 and $935 \mathrm{~cm}^{3}$, respectively, who received an SBRT dose of 45.5 Gy with an MLD of 17.08 Gy, experienced classical RILD. Among the two patients who experienced non-classical RILD, one patient was treated with an SBRT dose of 32.5 Gy and MLD of 15.26 Gy and had an ITV and uninvolved liver volume of 115 and $809 \mathrm{~cm}^{3}$, respectively. The other patient was treated with an SBRT dose of 40 Gy and MLD of 14.95 Gy and had an ITV and uninvolved liver volume of 311 and $986 \mathrm{~cm}^{3}$, respectively. Fifteen patients (55.6\%) had a CPS change of $\geq 2$; five $(33.3 \%)$ of them occurred in the first 3 months after SBRT.

There was no difference in the local tumor control rate when comparing lipiodol- and non-lipiodol-stained lesions (odds ratio, $0.40 ; 95 \% \mathrm{Cl}, 0.11-1.43$ ). 


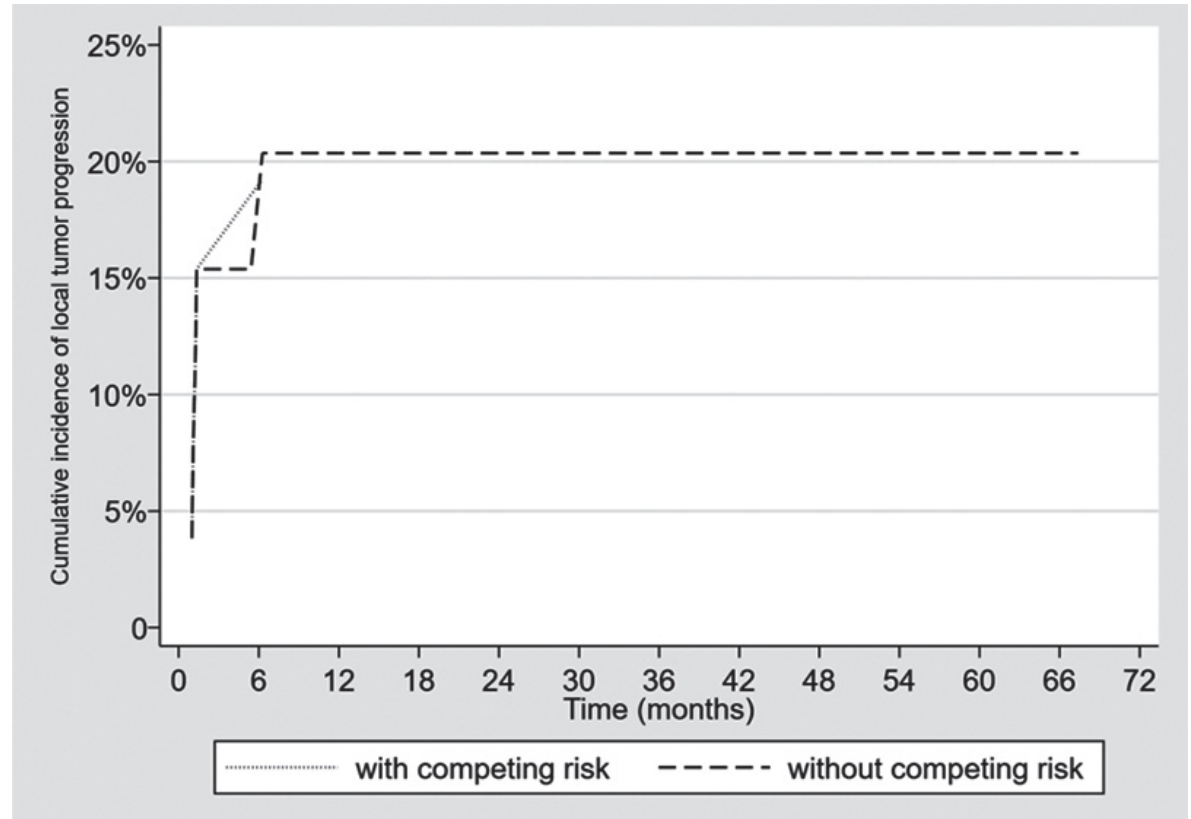

Figure 1 Cumulative incidence curve of SBRT-treated lesion progressiveness by competing risk analysis (any cause of death as a competing event)
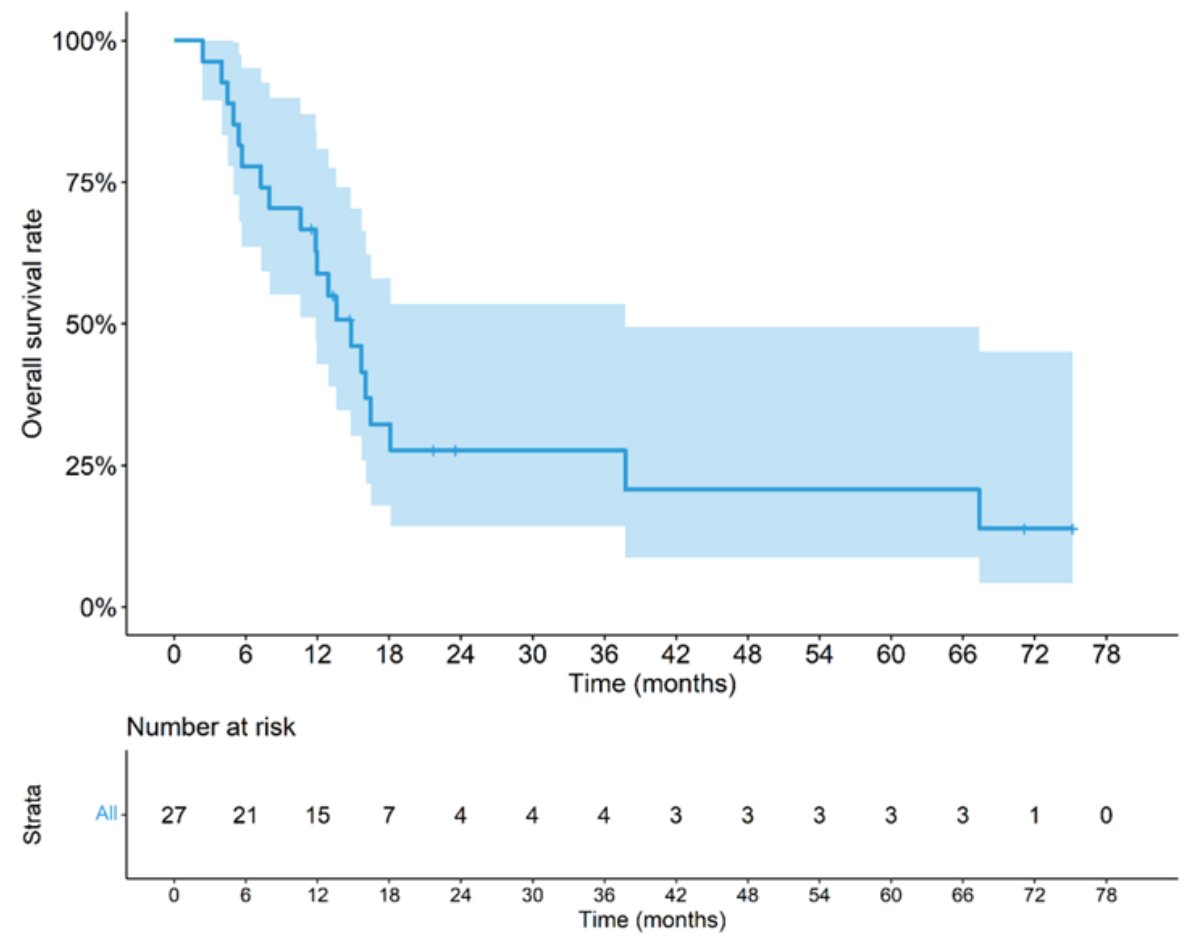

Figure 2 Kaplan-Meier curve for overall survival rate, with a 95\% confidence interval (shaded area) 

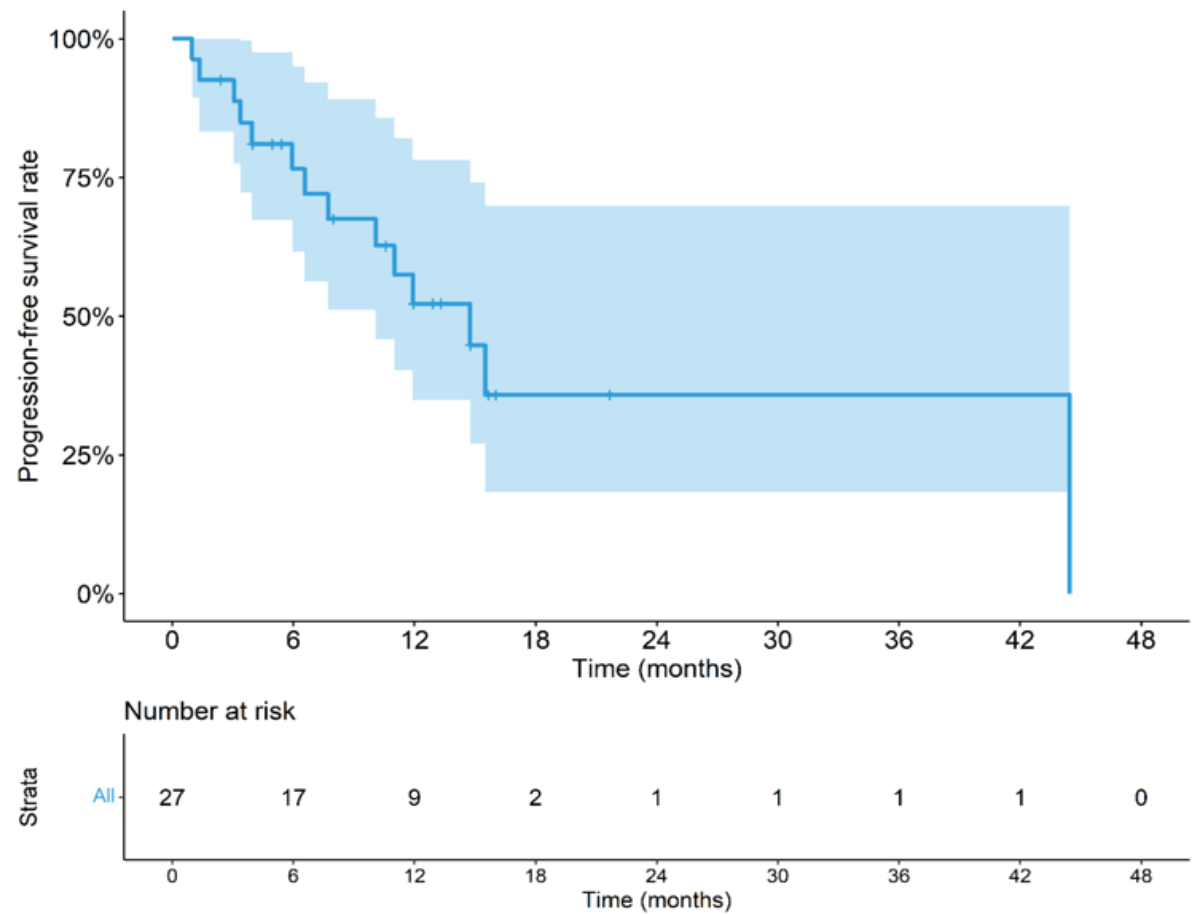

Figure 3 Kaplan-Meier curve for progression-free survival rate, with a 95\% confidence interval (shaded area)

Table 2 SBRT related acute complications [Grade (Gr.), \%]

\begin{tabular}{|c|c|c|c|c|c|c|}
\hline Term & & Baseline & $1^{\text {st }}$ week & $2^{\text {nd }}$ week & 1 month & 3 months \\
\hline \multirow[t]{5}{*}{ General } & Gr.0 & 96.3 & 88.0 & 88.0 & 72.0 & 76.0 \\
\hline & Gr.1 & 0 & 12.0 & 12.0 & 24.0 & 16.0 \\
\hline & Gr.2 & 3.7 & 0 & 0 & 0 & 0 \\
\hline & Gr.3 & 0 & 0 & 0 & 4.0 & 8.0 \\
\hline & Gr.4 & 0 & 0 & 0 & 0 & 0 \\
\hline \multirow[t]{5}{*}{ GI } & Gr.0 & 92.6 & 56.0 & 68.0 & 56.0 & 72.0 \\
\hline & Gr.1 & 0 & 44.0 & 28.0 & 36.0 & 16.0 \\
\hline & Gr.2 & 7.4 & 0 & 4.0 & 8.0 & 4.0 \\
\hline & Gr.3 & 0 & 0 & 0 & 0 & 8.0 \\
\hline & Gr.4 & 0 & 0 & 0 & 0 & 0 \\
\hline \multirow[t]{5}{*}{ Liver } & Gr.0 & 100.0 & 96.0 & 96.0 & 91.7 & 95.8 \\
\hline & Gr.1 & 0 & 4.0 & 4.0 & 8.3 & 4.2 \\
\hline & Gr.2 & 0 & 0 & 0 & 0 & 0 \\
\hline & Gr.3 & 0 & 0 & 0 & 0 & 0 \\
\hline & Gr.4 & 0 & 0 & 0 & 0 & 0 \\
\hline \multirow[t]{5}{*}{ Anemia } & Gr.0 & 44.4 & 52.6 & 50.0 & 47.6 & 45.8 \\
\hline & Gr.1 & 44.4 & 36.8 & 40.0 & 42.9 & 45.8 \\
\hline & Gr.2 & 3.7 & 5.3 & 10.0 & 9.5 & 8.3 \\
\hline & Gr.3 & 7.4 & 5.3 & 0 & 0 & 0 \\
\hline & Gr.4 & 0 & 0 & 0 & 0 & 0 \\
\hline
\end{tabular}


Table 2 (continued)

\begin{tabular}{|c|c|c|c|c|c|c|}
\hline Term & & Baseline & $1^{\text {st }}$ week & $2^{\text {nd }}$ week & 1 month & 3 months \\
\hline \multirow[t]{5}{*}{ Anemia } & Gr.0 & 44.4 & 52.6 & 50.0 & 47.6 & 45.8 \\
\hline & Gr.1 & 44.4 & 36.8 & 40.0 & 42.9 & 45.8 \\
\hline & Gr.2 & 3.7 & 5.3 & 10.0 & 9.5 & 8.3 \\
\hline & Gr.3 & 7.4 & 5.3 & 0 & 0 & 0 \\
\hline & Gr.4 & 0 & 0 & 0 & 0 & 0 \\
\hline \multirow[t]{5}{*}{ WBC } & Gr.0 & 66.7 & 42.1 & 45.0 & 52.4 & 41.7 \\
\hline & Gr.1 & 22.2 & 36.8 & 45.0 & 38.1 & 37.5 \\
\hline & Gr.2 & 7.4 & 21.1 & 10.0 & 9.5 & 16.7 \\
\hline & Gr.3 & 3.7 & 0 & 0 & 0 & 4.2 \\
\hline & Gr.4 & 0 & 0 & 0 & 0 & 0 \\
\hline \multirow[t]{5}{*}{ Platelets } & Gr.0 & 33.3 & 26.3 & 40.0 & 14.3 & 16.7 \\
\hline & Gr.1 & 44.4 & 42.1 & 35.0 & 38.1 & 45.8 \\
\hline & Gr.2 & 18.5 & 26.3 & 20.0 & 47.6 & 25.0 \\
\hline & Gr.3 & 3.7 & 5.3 & 5.0 & 0 & 12.5 \\
\hline & Gr.4 & 0 & 0 & 0 & 0 & 0 \\
\hline \multirow[t]{5}{*}{ INR } & Gr.0 & 69.2 & 57.1 & 66.7 & 61.1 & 47.8 \\
\hline & Gr.1 & 30.8 & 42.9 & 33.3 & 38.9 & 39.1 \\
\hline & Gr.2 & 0 & 0 & 0 & 0 & 8.7 \\
\hline & Gr.3 & 0 & 0 & 0 & 0 & 4.3 \\
\hline & Gr.4 & 0 & 0 & 0 & 0 & 0 \\
\hline \multirow[t]{5}{*}{ Bil } & Gr.0 & 66.7 & 45.8 & 58.3 & 60.9 & 50.0 \\
\hline & Gr.1 & 18.5 & 37.5 & 25.0 & 17.4 & 7.7 \\
\hline & Gr.2 & 14.8 & 12.5 & 16.7 & 21.7 & 34.6 \\
\hline & Gr.3 & 0 & 4.2 & 0 & 0 & 7.7 \\
\hline & Gr.4 & 0 & 0 & 0 & 0 & 0 \\
\hline \multirow[t]{5}{*}{ AST } & Gr.0 & 22.2 & 33.3 & 37.5 & 26.1 & 23.1 \\
\hline & Gr.1 & 66.7 & 57.1 & 54.2 & 65.2 & 61.5 \\
\hline & Gr.2 & 7.4 & 0 & 8.3 & 8.7 & 11.5 \\
\hline & Gr.3 & 3.7 & 9.5 & 0 & 0 & 3.8 \\
\hline & Gr.4 & 0 & 0 & 0 & 0 & 0 \\
\hline \multirow[t]{5}{*}{ ALT } & Gr.0 & 51.9 & 57.1 & 62.5 & 65.2 & 61.5 \\
\hline & Gr.1 & 40.7 & 33.3 & 33.3 & 30.4 & 38.5 \\
\hline & Gr.2 & 7.4 & 4.8 & 4.2 & 4.3 & 0 \\
\hline & Gr.3 & 0 & 4.8 & 0 & 0 & 0 \\
\hline & Gr.4 & 0 & 0 & 0 & 0 & 0 \\
\hline \multirow[t]{5}{*}{ ALP } & Gr.0 & 48.1 & 52.4 & 56.5 & 56.5 & 46.2 \\
\hline & Gr.1 & 48.1 & 42.9 & 39.1 & 34.8 & 50.0 \\
\hline & Gr.2 & 3.7 & 4.8 & 4.3 & 4.3 & 3.8 \\
\hline & Gr.3 & 0 & 0 & 0 & 4.3 & 0 \\
\hline & Gr.4 & 0 & 0 & 0 & 0 & 0 \\
\hline
\end{tabular}

${ }^{\dagger}$ Due to rounding of decimals, totals can add up to be less or greater than $100 \%$

ALP=alkaline phosphatase increased, ALT=alanine aminotransferase increased, AST=aspartate aminotransferase increased, Bil=blood bilirubin increased, $\mathrm{Gl}=$ gastrointestinal disorders, INR=international normalized ratio increased, WBC=white blood cell decreased 
Table 3 Details of General, Gastro-intestinal, and liver disorders [Grade (Gr.), \%]

\begin{tabular}{|c|c|c|c|c|c|c|}
\hline Term & Gr. & Base line & $1^{\text {st }}$ week & $2^{\text {nd }}$ week & 1 month & 3 months \\
\hline \multirow[t]{3}{*}{ Gen } & 1 & & Fatigue 12.0 & $\begin{array}{l}\text { Fatigue } 12.0 \\
\text { Malaise } 4.0\end{array}$ & $\begin{array}{l}\text { Fatigue } 16.0 \\
\text { Flu like } 4.0 \\
\text { Other } 8.0\end{array}$ & $\begin{array}{l}\text { Fatigue } 12.0 \\
\text { Limb edema } 4.0\end{array}$ \\
\hline & 2 & Fatigue 3.7 & & & & \\
\hline & 3 & & & & Flu like 4.0 & $\begin{array}{l}\text { Fatigue } 4.0 \\
\text { Flu like } 4.0 \\
\text { Limb edema } 4.0\end{array}$ \\
\hline \multirow[t]{3}{*}{$\mathrm{Gl}$} & 1 & Ascites 3.7 & $\begin{array}{l}\text { Nausea } 24.0 \\
\text { Dyspepsia } 8.0 \\
\text { Abd.distension } 4.0 \\
\text { Abd.pain } 4.0 \\
\text { Anorexia } 4.0 \\
\text { Ascites } 4.0\end{array}$ & $\begin{array}{l}\text { Nausea } 12.0 \\
\text { Abd.pain } 8.0 \\
\text { Dyspepsia } 8.0 \\
\text { Abd.distension } 4.0 \\
\text { Anorexia } 4.0 \\
\text { Ascites } 4.0\end{array}$ & $\begin{array}{l}\text { Dyspepsia } 24.0 \\
\text { Nausea } 12.0 \\
\text { Abd.distension } 4.0 \\
\text { Abd.pain } 4.0 \\
\text { Ascites } 4.0 \\
\text { Bloating } 4.0 \\
\text { Hematemesis } 4.0\end{array}$ & $\begin{array}{l}\text { Abd.distension } 8.0 \\
\text { Nausea } 8.0 \\
\text { Abd.pain } 4.0 \\
\text { Ascites } 4.0 \\
\text { Bloating } 4.0 \\
\text { Dyspepsia } 4.0\end{array}$ \\
\hline & 2 & $\begin{array}{l}\text { Ascites } 3.7 \\
\text { Nausea } 3.7\end{array}$ & & Dyspepsia 4.0 & $\begin{array}{l}\text { Abd.pain } 4.0 \\
\text { Nausea } 4.0\end{array}$ & Abd.distension 4.0 \\
\hline & 3 & & & & & $\begin{array}{l}\text { Ascites } 8.0 \\
\text { Nausea } 4.0\end{array}$ \\
\hline \multirow[t]{3}{*}{ Liver } & 1 & & Hepatic pain 4.0 & Hepatic pain 4.0 & Hepatic pain 8.0 & Hepatic pain 4.2 \\
\hline & 2 & & & & & \\
\hline & 3 & & & & & \\
\hline
\end{tabular}

†One patient may have more than one symptom in the same system Abd=abdominal, Gen=general disorders, Gl=gastrointestinal disorders

Table 4 Univariable analysis of overall survival

\begin{tabular}{|c|c|c|c|c|c|c|}
\hline Baseline characteristics & $\begin{array}{l}\text { Crude HR } \\
\text { for OS }\end{array}$ & $95 \% \mathrm{Cl}$ & $\begin{array}{l}\text { Adjusted HR } \\
\text { for OS }\end{array}$ & $95 \% \mathrm{Cl}$ & $\begin{array}{l}\text { p-value } \\
\text { (Wald) }\end{array}$ & $\begin{array}{l}p \text {-value } \\
\text { (LR) }\end{array}$ \\
\hline Gender (Ref=Male) & 1.14 & $0.24-4.96$ & 1.30 & $0.29-5.83$ & 0.732 & 0.740 \\
\hline Cause $(\mathrm{Ref}=\mathrm{HBV})$ & & & & & & 0.918 \\
\hline $\mathrm{HCV}$ & 0.73 & $0.24-2.18$ & 0.83 & $0.27-2.55$ & 0.738 & \\
\hline Non-viral & 1.20 & $0.42-3.45$ & 1.42 & $0.47-4.25$ & 0.535 & \\
\hline Age $(\operatorname{Ref} \leq 65)$ & 0.66 & $0.26-1.68$ & 0.63 & $0.25-1.61$ & 0.336 & 0.332 \\
\hline Lipiodol stained (Ref=No) & 1.35 & $0.56-3.28$ & 1.29 & $0.53-3.17$ & 0.577 & 0.580 \\
\hline BCLC stage at SBRT $(\operatorname{Ref}=A)$ & & & & & & 0.305 \\
\hline $\mathrm{B}$ & 0 & 0-infinity & 0 & 0-infinity & 0.998 & \\
\hline $\mathrm{C}$ & 1.38 & $0.18-10.54$ & 1.25 & $0.16-9.67$ & 0.833 & \\
\hline CPS at SBRT $($ Ref $=5)$ & & & & & & 0.099 \\
\hline 6 & 1.41 & $0.52-3.81$ & 1.47 & $0.54-4.00$ & 0.453 & \\
\hline 7 & 7.51 & $1.26-44.81$ & 6.06 & $0.97-38.06$ & 0.055 & \\
\hline 8 & 6.84 & $1.23-38.13$ & 8.02 & $1.39-46.42$ & 0.020 & \\
\hline SBRT dose & 0.97 & $0.90-1.04$ & 0.97 & $0.90-1.04$ & 0.379 & 0.378 \\
\hline AFP & 1.00 & $1.00-1.00$ & 1.00 & $1.00-1.00$ & 0.673 & 0.690 \\
\hline Uninvolved liver volume & 1.00 & $1.00-1.00$ & 1.00 & $1.00-1.00$ & 0.045 & 0.033 \\
\hline Mean liver dose & 1.15 & $1.00-1.31$ & 1.14 & $1.00-1.31$ & 0.073 & 0.056 \\
\hline Size & 1.09 & $0.96-1.24$ & 1.12 & $0.98-1.28$ & 0.091 & 0.114 \\
\hline ITV & 1.00 & $1.00-1.00$ & 1.00 & $1.00-1.00$ & 0.132 & 0.173 \\
\hline
\end{tabular}

$\mathrm{AFP}=$ alpha-fetoprotein, $\mathrm{BCLC}=$ Barcelona clinic liver cancer, $\mathrm{Cl}=$ confidence interval, $\mathrm{CPS}=\mathrm{Child}-\mathrm{P}$ ugh score, $\mathrm{HBV}=$ hepatitis $\mathrm{B}$ virus, $\mathrm{HCV}=$ hepatitis $\mathrm{C}$ virus, $\mathrm{HR}=$ hazards ratio, ITV=internal target volume, LR=likelihood ratio, OS=overall survival, Ref=reference, SBRT=stereotactic body radiotherapy 
Table 5 Univariable analysis of overall survival by uninvolved liver volume $\left(\mathrm{cm}^{3}\right)$

\begin{tabular}{|c|c|c|c|c|c|c|}
\hline $\begin{array}{l}\text { Stratified uninvolved } \\
\text { liver volume }\end{array}$ & $\begin{array}{l}\text { Crudes HR } \\
\text { for OS }\end{array}$ & $95 \% \mathrm{Cl}$ & $\begin{array}{l}\text { Adjusted HR } \\
\text { for OS }\end{array}$ & $95 \% \mathrm{Cl}$ & $\begin{array}{l}\text { p-value } \\
\text { (Wald) }\end{array}$ & $\begin{array}{l}\text { p-value } \\
\text { (LR) }\end{array}$ \\
\hline$>800($ Ref $\leq 800)$ & 0.49 & $0.06-3.86$ & 0.42 & $0.05-3.38$ & 0.416 & 0.466 \\
\hline$>1,000($ Ref $\leq 1,000)$ & 0.52 & $0.21-1.28$ & 0.40 & $0.15-1.08$ & 0.070 & 0.074 \\
\hline$>1,100($ Ref $\leq 1,100)$ & 0.47 & $0.19-1.16$ & 0.34 & $0.12-0.95$ & 0.039 & 0.039 \\
\hline$>1,200($ Ref $\leq 1,200)$ & 0.49 & $0.20-1.20$ & 0.51 & $0.21-1.28$ & 0.152 & 0.147 \\
\hline$>1,300($ Ref $\leq 1,300)$ & 0.38 & $0.14-1.08$ & 0.42 & $0.14-1.22$ & 0.110 & 0.097 \\
\hline$>1,400($ Ref $\leq 1,400)$ & 0.44 & $0.13-1.50$ & 0.48 & $0.14-1.70$ & 0.255 & 0.222 \\
\hline$>1,500($ Ref $\leq 1,500)$ & 0.33 & $0.08-1.44$ & 0.36 & $0.08-1.60$ & 0.178 & 0.130 \\
\hline$>1,600($ Ref $\leq 1,600)$ & 0.00 & 0-infinity & 0.00 & 0-infinity & 0.998 & 0.027 \\
\hline$>1,800($ Ref $\leq 1,800)$ & 0.00 & 0-infinity & 0.00 & 0-infinity & 0.998 & 0.027 \\
\hline
\end{tabular}

$\mathrm{Cl}=$ confidence interval, $\mathrm{HR}=$ hazards ratio, $\mathrm{LR}=$ likelihood ratio, $\mathrm{OS}=$ overall survival

Table 4 shows prognostic factors that affect overall survival. The average MLD of six patients who survived for less than 6 months was 14.5 Gy. The hazards ratio of patients who had an MLD of more than 14.5 Gy was 2.30 (95\% Cl, 0.94-5.60, p-value=0.067). All patients with RILD survived less than 6 months, and had an MLD of more than 14.5 Gy. A CPS of more than 7 was significantly correlated with poor overall survival.

Table 5 shows univariate analysis of overall survival by uninvolved liver volume. It was found that patients with an uninvolved liver volume $>1,100 \mathrm{~cm}^{3}$ had a significantly better overall survival. Three patients with RILD in this study had an uninvolved liver volume $<1,100 \mathrm{~cm}^{3}$.

\section{Discussion}

The local tumor control rate of the population in the current study was high, which was similar to that observed in many previous studies. ${ }^{83-26} \mathrm{~A}$ possible cause for this is that partially treated lesions may contain a lower burden of tumor cells than usual. ${ }^{27}$ We hypothesized that lipiodol-stained lesions could be treated more accurately than non-lipiodol-stained lesions, resulting in a higher local control rate, but we found no difference in the post hoc analysis.
Although, the local control rate was very high, the 1-year overall survival rate was low. Therefore, we investigated whether poor survival in the first 6 months after SBRT treatment was due to either the complications of treatment or poor disease status. When comparing the overall survival and progression-free survival rates, we found no significant difference during the first 6 months after SBRT. The 3-month overall survival and progression-free survival rates were $96.3 \%$ and $92.6 \%$, respectively; the 6-month overall survival and progression-free survival rates were $77.8 \%$ and $76.5 \%$, respectively. Thus, poor survival could have been due to poor disease status; however, there was also concern that patients experienced worsening liver function caused by SBRT. Therefore, factors that may cause hepatic complications, the MLD and uninvolved liver volume, were analyzed (Table 4 and 5). From the post hoc analysis, we could not exclude hepatic complications of SBRT from the possible causes of poor survival in the first 6 months after SBRT. Thus, our protocol should be modified to exclude patients with a CPS $>6$, and include only those patients with an uninvolved liver volume $>1,100$ $\mathrm{cm}^{3}$ for SBRT treatment. Moreover, only an MLD <14 Gy should be administered. 
We also investigated the prognostic factors of patients with cirrhosis caused by HBV. Surprisingly, the results we found (Table 4) - that most patients had HBV-did not significantly differ from those from countries with more advanced medical resources; although, the HBV-cause cirrhosis patients with HCC had a worse prognosis than from other causes. ${ }^{16,17}$ This could be due to differences in the patients' other baseline characteristics as well as the small number of patients in this study.

We observed more patients with abnormal liver function test results than those noted in previous studies ${ }^{8,9,24}$; however, the rate of RILD in this study (of $11.1 \%$ ) was similar to that seen in many previous studies..$^{23,28,29}$ This could be because our treatment protocol focused on the MLD. Hence, although the number of patients with abnormal liver function test results was high, the liver did not often progress to RILD. Meanwhile, the reason $12.5 \%$ of patients in this study experienced grade 3 adverse event of decreased platelet count at the three-month follow-up is unclear. However, there are several hypotheses; such as, a worsening of overall patient clinical conditions, splenic sequestration, bone marrow suppression, decreased plasma thrombopoietin, or platelet aggregation in the vessels around the tumor; due to radiation-induced endothelial cell injury. Therefore, radiation oncologists should be aware of the risk of decreased platelet counts when treating patients with a low platelet count at the start of SBRT.

When the median overall survival rate of HCC patients treated with SBRT and those treated with other treatment modalities in Thailand was compared, ${ }^{3,30}$ it was found that patients treated with SBRT in this study experienced a longer median overall survival than patients treated with transarterial oily chemoembolization (TOCE) or sorafenib; the median overall survival were 13.3, 6.3, and 6.3 months, respectively. Although, the median overall survival of patients treated with SBRT was more than twice that of those treated with other treatment modalities, the results could not be compared directly. This was because the time interval as well as baseline characteristics of the patients in each study differed. Additionally, patients suitable for SBRT must have an adequately preserved liver volume, while this is not a mandatory condition for those being treated with TOCE and sorafenib. However, SBRT has an advantage over TACE in HCC patients with main portal vein thrombosis, and that is the presence of a shunt, or no feeding artery. Nevertheless, this advantage should always be weighed against the risk of abnormal liver function and RILD. A randomized study or propensity score-matched study is suggested to identify the most beneficial treatment modality for this group of patients.

There are several limitations to this study. First, due to its retrospective nature, some data were incomplete or missing. Second, some of the acute SBRT-related toxicities recorded were intervened by treatment; such as, by blood transfusion or medication. However, this reflects real-life practice. For example, anemia cannot be left untreated when low hemoglobin levels are detected, and pain medication should be administered to patients experiencing pain. Thus, in some cases, the grading of acute adverse events may be lower than they actually are. Lastly, our study included a small cohort of patients; thus, some results; especially those of the univariate analysis, could be inaccurate. Therefore, our results should be interpreted and applied with caution.

To our knowledge, this is the first report regarding the efficacy and acute adverse events of SBRT-treated HCC patients in Thailand. One possible reason for more HCC patients not having received SBRT in the past is the concern of liver toxicity induced by SBRT. In addition to assessing the efficacy of SBRT, this study aimed to elucidate the details of acute adverse events following SBRT. The results of this study suggest that SBRT is both safe and effective in a specifically selected subset of patients, and recommends it be used more often in clinical practice. 


\section{Conclusion}

With a careful selection of patients, SBRT can be an effective local treatment for patients with HCC.

\section{Acknowledgement}

We would like to express my deep and sincere gratitude to Prof. Tippawan Liabsuetrakul, Ph.D., Ms. Nannapat Pruphetkaew, Prof. Virasakdi Chongsuvivatwong, Ph.D., and Alan Geater, Ph.D. from the Department of Epidemiology, Faculty of Medicine, Prince of Songkla University, for providing invaluable suggestions from the beginning to the end of this study. Also, Assoc. Prof. Kullathorn Thephamongkhol, from the Division of Radiation Oncology, Faculty of Medicine Siriraj Hospital, Mahidol University, for the suggestion and computation of the competing risk analysis.

\section{References}

1. Imsamran W. Cancer in Thailand Vol.IX, 2013-2015 [monograph on the Internet]. Bangkok: New Thammada Press; 2018. Available from: http://www.nci.go.th/th/File_download/Nci\%20 Cancer\%20Registry/In\%20Cancer\%20in\%20Thailand \%20IX\%200K.pdf

2. Omata M, Dan Y, Daniele B, Plentz R, Rudolph KL, Manns M, et al. Clinical features, etiology, and survival of hepatocellular carcinoma among different countries. J Gastroenterol Hepatol 2002;17(Suppl):S40-9.

3. Sithinamsuwan P. Review of 336 patients with hepatocellular carcinoma at Songklanagarind Hospital. World J Gastroenterol 2000;6:339.

4. Belghiti J, Kianmanesh R. Surgical treatment of hepatocellular carcinoma. HPB 2005;7:42-9.

5. Llovet JM, Bruix J. Systematic review of randomized trials for unresectable hepatocellular carcinoma: chemoembolization improves survival. Hepatol Baltim Md 2003;37:429-42.

6. Jun BG, Kim SG, Kim YD, Cheon GJ, Han KH, Yoo JJ, et al. Combined therapy of transarterial chemoembolization and stereotactic body radiation therapy versus transarterial chemoembolization for $\leq 5 \mathrm{~cm}$ hepatocellular carcinoma: propensity score matching analysis. PloS One 2018;13:e0206381.
7. Llovet JM, Ricci S, Mazzaferro V, Hilgard P, Gane E, Blanc JF, et al. Sorafenib in advanced hepatocellular carcinoma. N Engl J Med 2008;359:378-90.

8. Bujold A, Massey CA, Kim JJ, Brierley J, Cho C, Wong RKS, et al. Sequential Phase I and II Trials of Stereotactic Body Radiotherapy for Locally Advanced Hepatocellular Carcinoma. J Clin Oncol 2013;31:1631-9.

9. Andolino DL, Johnson CS, Maluccio M, Kwo P, Tector AJ, Zook $J$, et al. Stereotactic body radiotherapy for primary hepatocellular carcinoma. Int J Radiat Oncol Biol Phys 2011;81:e44753.

10. Lasley FD, Mannina EM, Johnson CS, Perkins SM, Althouse S, Maluccio $\mathrm{M}$, et al. Treatment variables related to liver toxicity in patients with hepatocellular carcinoma, Child-Pugh class A and $B$ enrolled in a phase 1-2 trial of stereotactic body radiation therapy. Pract Radiat Oncol 2015;5:e443-9.

11. Lax I, Blomgren H, Näslund I, Svanström R. Stereotactic radiotherapy of malignancies in the abdomen. Methodological aspects. Acta Oncol Stockh Swed 1994;33:677-83.

12. Song CW, Glatstein E, Marks LB, Emami B, Grimm J, Sperduto PW, et al. Biological Principles of Stereotactic Body Radiation Therapy (SBRT) and Stereotactic Radiation Surgery (SRS): Indirect Cell Death. Int J Radiat Oncol Biol Phys 2021;110:21-34.

13. Yang JD, Roberts LR. Hepatocellular carcinoma: a global view. Nat Rev Gastroenterol Hepatol 2010;7:448-58.

14. Sooklim K, Sriplung H, Piratvisuth T. Histologic subtypes of hepatocellular carcinoma in the southern Thai population. Asian Pac J Cancer Prev APJCP 2003;4:302-6.

15. Tangkijvanich $\mathrm{P}$, Hirsch $\mathrm{P}$, Theamboonlers A, Nuchprayoon I, Poovorawan Y. Association of hepatitis viruses with hepatocellular carcinoma in Thailand. J Gastroenterol 1999;34:227-33.

16. Li H, Qin $Y$, Li X, Li Q. Comparison of prognosis of small hepatocellular carcinoma patients with hepatitis B virus infection versus hepatitis C virus infection. Zhonghua Gan Zang Bing Za Zhi Zhonghua Ganzangbing Zazhi Chin J Hepatol 2009;17:426-8.

17. Abbas Z, Siddiqui A-R, Luck NH, Hassan M, Mirza R, Naqvi A, et al. Prognostic factors of survival in patients with nonresectable hepatocellular carcinoma: hepatitis C versus miscellaneous etiology. JPMA J Pak Med Assoc 2008;58:602-7.

18. Oken MM, Creech RH, Tormey DC, Horton J, Davis TE, McFadden ET, et al. Toxicity and response criteria of the Eastern Cooperative Oncology Group. Am J Clin Oncol 1982;5:649-55. 
19. Laura A. Dawson. Radiation Therapy Oncology Group RTOG 1112 Randomized phase III study of Sorafenib versus stereotactic body radiation therapy followed by Sorafenib in hepatocellular carcinoma [monograph on the Internet]. Philadelphia: RTOG foundation. Available from: https://www.rtog.org/Clinical Trials/ProtocolTable/StudyDetails. aspx? ptid=387\&mode= broadcasts\&page $896=3 \&$ study $=1112$

20. Lencioni R, Llovet JM. Modified RECIST (mRECIST) assessment for hepatocellular carcinoma. Semin Liver Dis 2010;30:52-60.

21. Eisenhauer EA, Therasse P, Bogaerts J, Schwartz LH, Sargent D Ford $\mathrm{R}$, et al. New response evaluation criteria in solid tumours: revised RECIST guideline (version 1.1). Eur J Cancer Oxf Engl 1990;45:228-47.

22. Common Terminology Criteria for Adverse Events (CTCAE) Version 5.0 [monograph on the Internet]. Maryland: U.S. Department of Health and Human services; 2017. Available from: https://ctep.cancer.gov/protocoldevelopment/electronic_ applications/docs/CTCAE_v5_Quick_Reference_5x7.pdf

23. Tse RV, Hawkins M, Lockwood G, Kim JJ, Cummings B, Knox J, et al. Phase I study of individualized stereotactic body radiotherapy for hepatocellular carcinoma and intrahepatic cholangiocarcinoma. J Clin Oncol 2008;26:657-64.

24. Chan LC, Chiu SKW, Chan SL. Stereotactic radiotherapy for hepatocellular carcinoma: report of a local single-centre experience. Hong Kong Med J Xianggang Yi Xue Za Zhi 2011; 17:112-8.
25. Dewas S, Mirabel X, Kramar A, Jarraya H, Lacornerie T, DewasVautravers $\mathrm{C}$, et al. Stereotactic body radiation therapy for liver primary and metastases: the Lille experience. Cancer Radiother J Soc Francaise Radiother Oncol 2012;16:58-69.

26. Bae SH, Kim M-S, Cho CK, Kim KB, Lee DH, Han CJ, et al. Feasibility and efficacy of stereotactic ablative radiotherapy for Barcelona Clinic Liver Cancer-C stage hepatocellular carcinoma. J Korean Med Sci 2013;28:213-9.

27. Ohri N, Tomé WA, Méndez Romero A, Miften M, Ten Haken RK, Dawson LA, et al. Local Control After Stereotactic Body Radiation Therapy for Liver Tumors. Int J Radiat Oncol Biol Phys 2021:110:188-95.

28. Méndez Romero A, Wunderink W, Hussain SM, De Pooter JA, Heijmen BJM, Nowak PCJM, et al. Stereotactic body radiation therapy for primary and metastatic liver tumors: a single institution phase i-ii study. Acta Oncol Stockh Swed 2006;45: 831-7.

29. Louis C, Dewas S, Mirabel X, Lacornerie T, Adenis A, Bonodeau $F$, et al. Stereotactic radiotherapy of hepatocellular carcinoma: preliminary results. Technol Cancer Res Treat 2010;9:479-87.

30. Chantharasamee J, Chotiyaputta W, Nimmannit A, Techawatanawanna S. Survival outcomes of metastatic/ unresectable hepatocellular carcinoma in patients treated with sorafenib. J Med Assoc Thai 2019;102:107-12. 